\title{
Arborescences
}

Revue d'études françaises

\section{Lire le texte et son espace Une introduction}

\section{Antje Ziethen, Caroline Lebrec et Janet Paterson}

Numéro 3, juillet 2013

Lire le texte et son espace : outils, méthodes, études

URI : https://id.erudit.org/iderudit/1017362ar

DOI : https://doi.org/10.7202/1017362ar

Aller au sommaire du numéro

Éditeur(s)

Département d'études françaises, Université de Toronto

ISSN

1925-5357 (numérique)

Découvrir la revue

Citer ce document

Ziethen, A., Lebrec, C. \& Paterson, J. (2013). Lire le texte et son espace : une introduction. Arborescences, (3). https://doi.org/10.7202/1017362ar

Tous droits réservés @ Département d'études françaises, Université de Toronto, Ce document est protégé par la loi sur le droit d’auteur. L’utilisation des 2013 services d’Érudit (y compris la reproduction) est assujettie à sa politique d'utilisation que vous pouvez consulter en ligne.

https://apropos.erudit.org/fr/usagers/politique-dutilisation/ 


\title{
LIRE LE TEXTE ET SON ESPACE
}

\section{Une introduction}

\author{
Antje Ziethen, Caroline Lebrec et Janet Paterson \\ Université de Toronto
}

L'espace de notre vie n'est ni continu, ni infini, ni homogène, ni isotrope. Mais sait-on précisément où il se brise, où il se courbe, où il se déconnecte et où il se rassemble ? On sent confusément des fissures, des hiatus, des points de friction, on a parfois la vague impression que ça se coince quelque part, ou que ça éclate, ou que ça se cogne. Nous cherchons rarement à en savoir davantage et le plus souvent nous passons d'un endroit à l'autre, d'un espace à l'autre sans songer à mesurer, à prendre en charge, à prendre en compte ces laps d'espace. Le problème n'est pas d'inventer l'espace, encore moins de le ré-inventer $[\ldots]$, mais de l'interroger, ou, plus simplement encore, de le lire [...].

Georges Perec, «Prière d'insérer », Espèces d'espaces, Paris, Galilée, coll. «L'espace critique », 2000 [1974].

Depuis la fin du $\mathrm{Xx}^{\mathrm{e}}$ siècle, la mondialisation et les phénomènes connexes tels que le time-space compression (Harvey 1989), la transmigration et le cyberespace ont profondément marqué notre vie quotidienne et le discours social. Les mouvements d'individus, de peuples, d'idées, d'objets et de capital à travers les frontières géographiques, linguistiques et nationales ont amené les chercheurs issus de disciplines diverses à repenser l'espace dans l'ère postmoderne, hypermoderne, surmoderne ou encore postcoloniale. La formation de nouvelles réalités spatiales a suscité une prolifération de notions théoriques incluant l'hétérotopie (Foucault 1984), le space of flows (Castells 1989), les non-lieux (Augé 1992), le tiers espace (Bhabha 1994), la postmetropolis (Soja 2000) ou encore le glocal. La mobilité des populations, leur densité croissante ainsi que les constellations sociales, culturelles et économiques qui en découlent soulèvent des questionnements pour les géographes, sociologues, anthropologues, urbanistes et philosophes. Le présent dossier s’inscrit dans le contexte actuel de cette réorientation des sciences sociales et humaines communément appelée le spatial turn (Warf et Arias 2009 : 1) qui a également influencé les études littéraires. L'émergence récente d'approches dites "géocentrées » qui conjuguent la théorie littéraire à la géographie, la sociologie, la philosophie, l'architecture et l'écologie témoigne de l'actualité du sujet proposé. Pour les chercheurs, lire le texte et son espace implique forcément de réfléchir au fonctionnement même du texte littéraire et à son 
rapport au monde, car l'espace-temps empirique traverse l'œuvre en véritable sédiment du processus poétique tout en étant reconfiguré par le verbe et l'imaginaire. Ansgar Nünning écrit à ce propos :

À partir de ses procédés narratifs, un roman organise les éléments sélectionnés et crée des mondes imaginaires dont ni les éléments spécifiques ni les relations que ceux-ci entretiennent entre eux ne produisent de simples calques d'espaces réels ou d'époques historiques. Il s'agit de modèles historiques et spatiaux qui ont été esthétiquement composés. (Nünning 2009 : 43 ; notre traduction).

Qui plus est, la transfiguration du réel par la fiction met en avant la pertinence de la littérature pour d'autres disciplines «spatiales », c'est-à-dire ayant l'espace comme objet d'étude (la géographie, l'urbanisme, etc.). Lorsque nous étudions l'espace en termes littéraires, il ne s'agit donc pas seulement d'assimiler les théories d'autres disciplines mais aussi de montrer ce que les études littéraires leur offrent en retour (Prieto 2011 : 13).

Comment donc appréhender, lire et interpréter l'espace fictionnel ? Quels nouveaux outils théoriques nous permettent d'en envisager l'immense potentiel ? Quelles sont ses fonctions au sein du texte littéraire ? La littérature peut-elle contribuer à la construction des espaces référentiels ? Ces interrogations se trouvent au cœur même de ce dossier d'Arborescences qui a pour objet de lire l'espace sous ses formes multiples : celle du «contexte géographique dans lequel le texte est produit ou auquel le texte se réfère », celle de «l'espace signifié par le texte», voire de l'organisation du monde fictionnel, ou encore celle de «l'espace physiquement occupé par le texte» (Ryan 2003 : 336 ; notre traduction). Par conséquent, les méthodes et outils d'analyse divergent. Certaines études traitent l'espace d'un point de vue géographique, tandis que d'autres l'appréhendent d'un point de vue narratologique, sémiotique ou encore historique. Étudiées de concert, ces déclinaisons sont nécessaires pour comprendre la façon dont l'espace informe, et est informé par, le discours de l'auteur, le genre littéraire, la lecture et les modalités narratives. L’acte poétique tout entier est empreint de la composante spatiale — du contenu du texte à sa structure, du mode de production à la réception. Les parcours méthodologique et théorique privilégiés par nos contributeurs les ont conduit vers un champ d'étude encore peu fréquenté mais extrêmement fertile et souvent interdisciplinaire. Dans les articles de ce dossier, la convergence des savoirs est source de dialogue avec l'Autre. Elle génère en effet de nouvelles connaissances et nous offre des éléments de réponse aux questions brûlantes qui définissent nos sociétés contemporaines.

En guise d'entrée en matière à la première partie du dossier intitulée « Théorie et praxis », la contribution d'Antje Ziethen présente les différentes approches littéraires " géocentrées » qui ont été formalisées, consolidées ou renouvelées dans le sillage du remaniement épistémologique suscité par 
le spatial turn. Ziethen commence par une revue des recherches qui ont établi les bases théoriques d'une pensée spatiale en littérature et se tourne ensuite vers les études plus récentes. En premier lieu, sont abordés les travaux de Mikhail Bakhtine, Youri Lotman, Henri Mitterand et Jean Weisgerber afin d'exposer, en deuxième lieu, la narratologie de l'espace, les romans-géographes, la géocritique, la géographie de la littérature, la géopoétique, la pensée-paysage et l'écocritique. Ces prolégomènes positionnent le présent recueil dans un contexte scientifique précis tout en défrichant les grands axes des contributions présentées par la suite. À leur tour, Rachel Bouvet et Myriam Marcil-Bergeron ainsi que Richard Spavin poursuivent la réflexion théorique sur deux des approches abordées: la géopoétique et la géocritique. Rachel Bouvet et Myriam Marcil-Bergeron démontrent comment la géopoétique - champ de recherche et de création transdisciplinaire - permet de jeter un regard différent sur le récit de voyage. Elles exposent d'abord certains principes fondamentaux — le nomadisme, le dehors et le rapport à la terre — de cette théorie située à la croisée de la littérature et de la géographie. L'analyse des Écrits sur le sable d'Isabelle Eberhardt illustre ensuite en quoi consiste cette méthode qui mobilise les notions de paysage, de polysensorialité et de mouvement. Richard Spavin, quant à lui, met la géocritique de Bertrand Westphal en relation avec la théorie des climats de Jean Bodin. Cette conjonction originale de deux approches très différentes et séparées temporellement permet à l'auteur d'identifier des préoccupations similaires. En effet, Bodin et Westphal promeuvent une vision hybride du monde qui passe par une instrumentalisation rhétorique de l'espace.

Dans la deuxième partie du dossier intitulé « Géographies imaginaires », Robin Craig et Nicholas Serruys explorent, respectivement, les géographies imaginaires du roman gothique au tournant du XIX ${ }^{\mathrm{e}}$ siècle et de l'uchronie québécoise dans l'objectif de démontrer comment le genre littéraire et ses chronotopes cristallisent les préoccupations d'une époque spécifique. Dans son article, Robin Craig prend pour objet le roman Malvina de Sophie Cottin afin d'y étudier la fonction et la signification du château. Ce dernier est un espace hybride et interstitiel qui est emblématique de la transition du libertinage pendant le siècle des Lumières à celui du Romantisme, comme le montrent les figures érotisantes, telles le boudoir, le décor pictural évocateur de scènes frivoles, les fenêtres renvoyant sur l'espace érotisé du château au lieu de renvoyer sur le monde extérieur, tout ceci au service d'une maittresse abusive qui inspire la crainte. Nicholas Serruys, pour sa part, se consacre à un genre encore peu présent dans les études géocentrées — l'uchronie, sous-genre de la science fiction. S'appuyant sur Les Voyageurs malgré eux de l'auteure québécoise Élisabeth Vonarburg, l'auteur sonde les mécanismes littéraires qui produisent un écart entre l'espace-temps factuel et fictif du Québec. 
Dans ce roman, la mise en scène d'une migration interdimensionnelle et de mondes parallèles appelle à une réévaluation des enjeux historiques et territoriaux propres à la société québécoise.

Dans la troisième partie intitulée «Configurations urbaines », les contributions de Caroline Lebrec et Andrée Mélissa Ferron se tournent vers un des grands topoï du roman contemporain : la ville. Dans une étude consacrée à la représentation de la ville d'un auteur oulipien, Ian Monk, Lebrec montre comment l'espace de la ville contemporaine de banlieue reflète une vision urbaine du sale, du déchet, du rejet, à travers un portrait pluriel d'habitants humiliés et anonymisés. L'ancrage dans l'espace renvoie vers l'identitaire et non pas dans le sociologique, car l'accent est mis sur le lieu où l'on vit, plutôt quesur celui d'où l'on vient ou sur la catégorie socio-culturelle à laquelle on appartient. Lebrec révèle également comment le portrait de la ville imaginaire de la banlieue française s'inscrit à l'encontre du rayonnement culturel d'une « francitude » de carte postale pour s'ancrer dans un réel suintant la violence et le dégoût (Roubaud 2007 : 13). De son côté, Andrée Mélissa Ferron amène le lecteur dans le Moncton du poète Gérald Leblanc pour explorer le rapport entre texte, corps et espace urbain. Adoptant les allures d'un personnage, la ville s'impose comme médiatrice d'une identité acadienne multiple et dynamique libérée des contraintes du passé et du folklore. Prenant corps par le langage et le fantasme, le Moncton de Leblanc se défait de ses attributs d'espace cloîtré et oppressant afin de se muer en une béance sur le continent et sur l'Autre. L'Acadie se dessine ici non pas dans les traits traditionnels d'une collectivité monolithique mais par une polyphonie de subjectivités.

Dans la quatrième partie de notre dossier intitulée "Textualités géographiques », Isabelle Chol, Tara Collington et Annabelle Seoane examinent l'espace à la fois d'un point de vue thématique et formel en abordant la poésie graphique, l'intermédialité et le discours des guides touristiques. À partir d'une lecture des recueils d'Emmanuel Hocquard et de Jean-Michel Espitallier, Isabelle Chol analyse l'espace en tant que support et medium d'une parole qui se donne non seulement à lire mais également à voir. En géomètre et géographe, le poète organise la graphie pour créer des taches, des trous, des lignes dont le sens dépasse les limites imposées par la page et les mots. L'espace scriptural se transforme ainsi en un lieu d'expérimentation ludique sur la matérialité de la poésie et sur sa spécificité à représenter le monde, lieu qui permet de déconstruire les structures et hiérarchies habituelles. De son côté, Tara Collington s’interroge sur les dispositifs spatiaux dans le récit Le Disparu de Salonique d'Aliette Armel, qui est illustré de 25 photographies prises par le grand-père de l'auteure. Ici, l'espace fictionnel procède à la fois du verbe et de l'image dont les modalités de représentations divergent. Le recours à l'intermédialité est source de confusion pour le lecteur. Il inscrit le texte dans 
un discours sur la guerre par un jeu de voilement/dévoilement de photographies d'espionnage donnant ainsi un éclairage au récit dans lequel la dimension de l'espionnage n'est pas lisible. Qui plus est, la démarche d'Armel produit un corps à la fois textuel et iconographique, qui soulève la question du rapport entre mimèsis et poï̀sis. Enfin, en guise de clôture de notre dossier, Annabelle Seoane mobilise les outils de l'analyse du discours dans l'objectif de scruter les textes touristiques sur le Cambodge et d'exposer la façon dont ils produisent un espace « guidé ». L'auteure dévoile de la sorte les maintes stratégies du Guide Gallimard et du Guide du Routard qui rendent l'espace référentiel accessible et utile aux lecteurs, notamment par la fragmentation, l'hyperstructuration et la sélection d'informations données dans un mode propre à chacun des guides. La présente collection d'articles originaux et diversifiés apporte, nous en sommes convaincues, un éclairage sur les recherches actuelles en littérature française et francophone en mettant au jour la fonction fondamentale de l'espace fictionnel, les modalités de sa production et, par extension, la capacité du texte littéraire non seulement de représenter mais aussi d'informer la manière dont nous percevons le monde.

\section{Références bibliographiques}

Augé, M. 1992. Non-lieux. Introduction à une anthropologie de la surmodernité. Paris : Seuil.

Bhabha, H.K. 1992. The Location of Culture. London; New York : Routledge.

Castells, M. 1989. The Informational City: Information, Technology, Economic Restructuring and the UrbanRegional Process. Oxford, UK, Cambridge, MA : Blackwell.

Foucault, M. 1984. «Des Espaces autres ». Architecture, Mouvement, Continuité, n5, 46-49.

Harvey, D. 1989. The Condition of Postmodernity: An Enquiry into the Origins of Cultural Change. Oxford, Mass. : Blackwell.

Nünning, A. 2009. «Formen und Funktionen literarischer Raumdarstellung : Grundlagen, Ansätze, narratologische Kategorien und neue Perspectiven ». W. Hallet et B. Neumann (dirs.). Raum und Bewegung in der Literatur. Die Literaturwissenschaften und der Spatial Turn. Bielefeld : Transcript Verlag. 33-52.

Perec, G. 2000 [1974]. « Prière d'insérer ». Espèces d'espaces. Paris : Galilée.

Prieto, E. 2011. « Geocriticism, Geopoetics, Geophilosophy, and Beyond ». R. T. Tally (dir.). Geocritical Explorations: Space, Place, and Mapping in Literary and Cultural Studies. New York: Palgrave MacMillan. 13-28.

Roubaud, J. 2007. «Ian Monk traverse le channel». Plonk Town. Paris : Cambourakis.

Ryan, M.-L. 2003. « Narrative Cartography: Toward a Visual Narratology ». T. Kindt et H.-H. Müller (dirs.). What is Narratology? Questions and Answers Regarding the Status of a Theory. Berlin; New York: Walter de Gruyter. 333-364. 
Soja, E.W. 2000. Postmetropolis. Critical Studies of Cities and Regions. Oxford, UK ; Malden, MA : Blackwell Publishers.

Warf, B. et S. Arias. 2009. "Introduction: The Reinsertion of Space into the Social Sciences and Humanities ». B. Warf et S. Arias (dirs.). The Spatial Turn: Interdisciplinary Perspectives. London : Routledge. 1-10. 